\section{Mucinous eccrine carcinoma of the eyelid: re-emphasizing the need for awareness of rare lesions}

Kiran Krishne Gowda, Parimal Agarwal, Amanjit Bal

Department of Histopathology, Post Graduate Institute of Medical Education and Research, Chandigarh, India

\section{Abstract}

We report here the case of a man presenting with mucinous eccrine carcinoma (MEC) involving eyelid. This is a rare adencocarcinoma of the skin that originates from the deepest portion of eccrine sweat duct. The aim of our paper is to underline the importance of distinguishing MEC from metastatic carcinomas of the skin, making clinicians aware that what seems to be a harmless benign lesion may be a malignant one.

\section{Introduction}

Mucinous eccrine carcinoma (MEC) is a rare form of adenocarcinoma of skin, first described by Mendoza and Helwigin 1971. ${ }^{1}$ The clinical appearance of this lesion is varied and can be in form slow-growing, solitary, asymptomatic, flesh-colored nodule or can have ulcerated lesion. Due to varied clinical appearance, the differential diagnoses ranges from benign to malignant lesions, thus requiring histopathological examination for diagnosis.

\section{Case Report}

A 56 year old man presented with a $10 \times 15$ mm smooth, bluish-red nodule over the right lower eyelid of 2 months duration. There was no extension into adjacent bony structures. His vision was intact. His physical examination did not reveal any lymphadenopathy and systemic examination was unremarkable. Imaging studies did not reveal any lesions in other organs of the body. With clinical diagnosis of benign adnexal tumor of eyelid, wide local excision of the lesion was performed with $5 \mathrm{~mm}$ margins. Histopathological examination showed a circumscribed tumor within the dermis, divided into numerous compartments by fibrous strands. The compartments showed tumor cells in nests, cords and few tubules with pools of extracellular mucin in the background (Figure 1). The tumor cells showed mild nuclear atypia with abundant amount of cytoplasmic mucin. The mucin (intra and extra-cellular) showed positive reactions with Periodic acid Schiff and was resistant to diastase. Alcian blue staining of mucinous material revealed strong positivity at $\mathrm{pH} 2.5$ and weak positivity at $\mathrm{pH} 0.4$ indicating that mucin is non-sulfated and represents sialomucin which represents epithelial mucin, suggesting an eccrine origin. ${ }^{2}$ Immunohistochemistry for Cytokeratin 7 was positive while, Cytokeratin 20 and Thyroid transcription factor-1 (TTF-1) were negative. Thus a diagnosis of mucinous eccrine carcinoma of the right eyelid was made. On 6 months follow up, patient does not have recurrence, any regional or distant metastasis. He is currently on annual control for early detection of recurrence, metastasisregional or distal.

\section{Discussion and Conclusions}

Mucinous eccrine carcinoma generally affects patients in their $60 \mathrm{~s}$, with a male:female ratio of $2: 1$. It is considered a proliferation of cells that originates from deepest portion of eccrine sweat duct. It is most commonly found on eyelid (38\%) but can also occur on face (20.3\%) and scalp (16\%). ${ }^{3}$ The clinical appearance is varied and can be in form of slow-growing, solitary, asymptomatic, flesh-colored nodule, similar to the present case or can have ulcerated lesion. The clinical differential diagnosis ranges from benign lesions like lipoma, neuroma and cutaneous cysts to malignant tumors like basal cell carcinoma, sebaceous carcinoma, melanoma and metastatic adenocarcinoma. Although clinical presentation is heterogeneous, histology of MEC is quite characteristic and distinct from above mentioned lesions aiding in its diagnosis. Its characteristic feature includes large pools of extracellular basophilic mucin, containing tumor cells with focal tubule formation indicating eccrine differentiation. ${ }^{2}$ The tumor cells are more atypical in metastatic type, which invade between collagen bundles at the margin of the nodule. However, its definitive diagnosis requires exclusion of metastatic visceral malignancy, especially from mucinous carcinoma breast, lung and colorectal tumors. It is important to differentiate this tumor from a metastatic mucinous adenocarcinoma which can be done by tissue-specific special stains and immunostains. MEC stains positive for periodic acid-Schiff, Alcian blue at $\mathrm{pH} 2.5$ and is negative at $\mathrm{pH} 0.4$ differentiating $\mathrm{MEC}$ from other sweat gland tumors and gastrointestinal
Correspondence: Amanjit Bal, Department of Histopathology, Post Graduate Institute of Medical Education and Research, Sector 12, Chandigarh, 160012, India.

Tel.: +91.172.2747.585.

E-mail: docaman5@hotmail.com

Key words: eyelid, mucinous eccrine carcinoma.

Contributions: KKG, PA, participated in writing the manuscript along with photography; $\mathrm{AB}$, participated in writing and editing the manuscript.

Conflict of interests: the authors declare no potential conflict of interests.

Received for publication: 29 May 2014.

Revision received: 2 August 2014.

Accepted for publication: 11 August 2014.

This work is licensed under a Creative Commons Attribution NonCommercial 3.0 License (CC BYNC 3.0).

(C)Copyright K.K. Gowda et al., 2014

Licensee PAGEPress, Italy

Dermatology Reports 2014; 6:5498

doi:10.4081/dr.2014.5498

neoplasms which contain sulfomucin rather than sialomucin. Mucinous eccrine carcinomas may be positive for cytokeratins (CK7, CAM5.2), carcinoembryonic antigen (CEA), epithelial membrane antigen (EMA), estrogen receptor (ER), progesterone receptor (PR), p63, mucous-associated peptides of the trefoil factor family (TFF1 and 3), tumor-associated glycoprotein (TAG-72).$^{4-6}$ Cytokeratin 20 stains most colorectal carcinomas while it is absent in MEC. Metastatic breast carcinomas also share staining characters with MEC making it difficult to rely solely on immunohistochemical stains. Adenocarcinomas of lung stain positively with TTF-1 while MEC do not stain with the antibody. Although immunohistochemical staining pattern can help differentiate MEC from metastatic tumors, it cannot be used with $100 \%$ certainty. A final diagnosis can be made by thorough clinical investigation and systemic imaging, which excludes presence of a more common primary mucinous carcinoma of lung, gastrointestinal tract and other sites, which was done in present case. Other important feature that helps in making diagnosis of MEC is its characteristic histology and histochemical staining as seen in current case. Because of rarity of MEC, there is neither a definite staging method nor standard guidelines for treatment. Surgery is the treatment of choice as mucinous eccrine carcinoma is resistant to radiotherapy and chemotherapy. Wide local excision with $5 \mathrm{~mm}$ margins is preferred. The high recurrence rate reported in literature is attributed to incomplete excision 

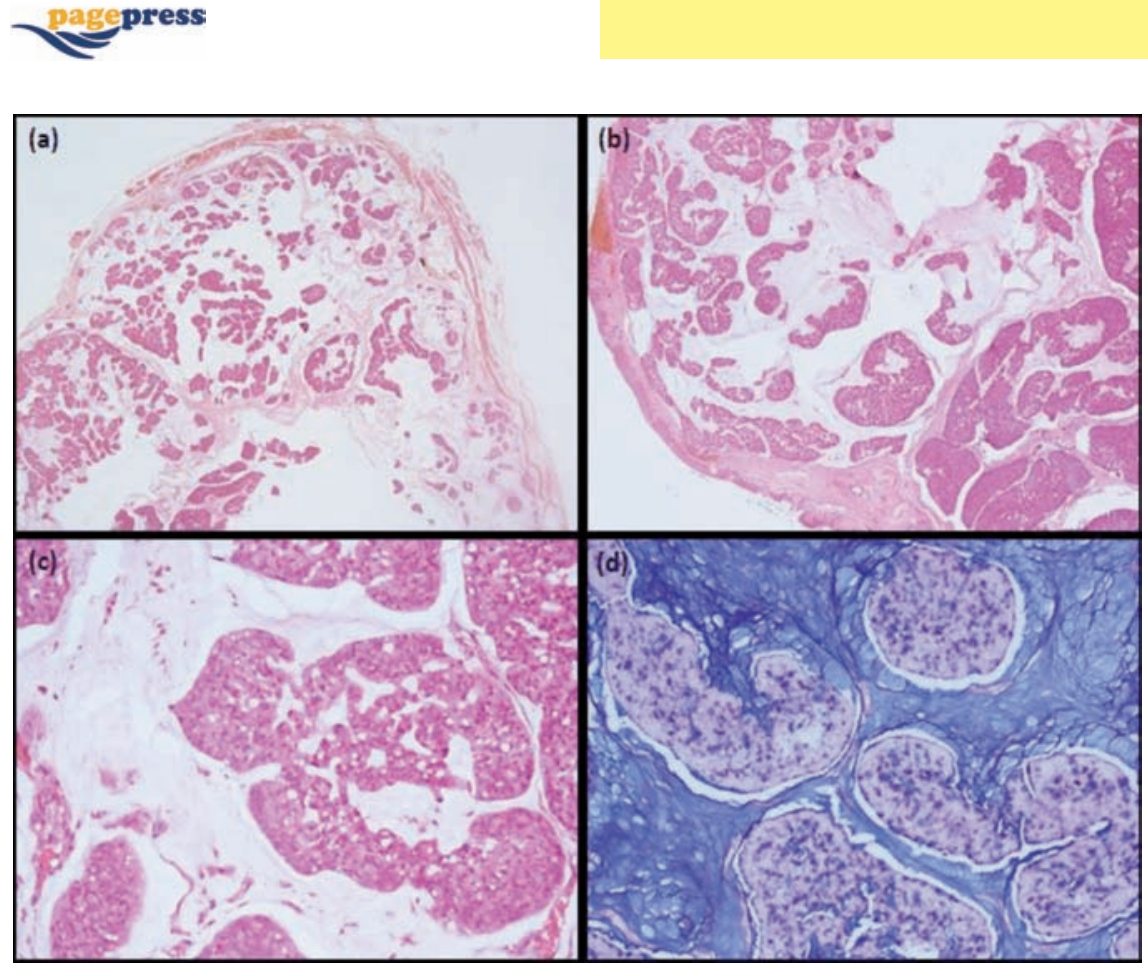

Figure 1. A well circumscribed tumor in the dermis, divided into numerous compartments by fibrous strands. Each compartment consists of tumor cells surrounded by abundant mucin (Hematoxylin \& Eosin: a, 10×; b, 20x). Focal tubules with lumen can also be noted (Hematoxylin \& Eosin: c, 40×). Alcian blue stain at pH 2.5 highlighting the mucin $(d, 40 \times)$.

of the tumor. In recurrent cases and sites such as eyelid, Moh's micrographic surgery (with 2 $\mathrm{mm}$ margins) is preferred as it ensures the complete removal of the tumor along with its margins, and is tissue preserving. Due to the rarity of this entity, the usefulness of sentinel lymph node biopsy and regional lymphadenectomy is not proven. ${ }^{6}$ However rarity of this tumor precludes comparative evaluation of treatment. Local recurrence rate of MEC varies from $0-33 \%$ in the literature. ${ }^{7,8}$ Metastasis to regional lymph node is rare (10\%) with distant metastasis in $3 \%$ of cases. Mortality has also been reported to be low
$(<2 \%)$ in previous published studies. ${ }^{7}$ In summary, it is important to distinguish MEC from metastatic carcinomas to skin. The high degree of differentiation, intercellular cohesion, limited angiogenesis and extensive mucin production, which shields tumor antigens are factors which prevent loco-regional and systemic metastasis. The clinicians should be aware that what appears to be a harmless benign lesion may be a malignant. Though indolent tumor it requires concerted effort from clinician, radiologist and pathologist in combination to make a definitive diagnosis of MEC.

\section{References}

1. Lennox B, Pearse AG, Richards HG. Mucin secreting tumors of the skin with special reference to the so-called mixed-salivary tumor of the skin and its relation to hidroadenoma. J Pathol Bacteriol 1952;64: 865-80.

2. Headington JT. Primary mucinous carcinoma of skin: histochemistry and electron microscopy. Cancer 1977;39:1055-63.

3. Snow SN, Reizner GT. Mucinous eccrine carcinoma of the eyelid. Cancer 1992;70:2099-104.

4. Carson HJ, Gattuso P, Raslan WF, Reddy V. Muinous carcinoma of the eyelid. An immunohistochemical study. Am J Dermatopathol 1995;17:494-8.

5. Zhang Q1, Wojno TH, Fitch SD, Grossniklaus HE. Mucinous eccrine adenocarcinoma of the eyelid: report of 6 cases. Can J Ophthalmol 2010;45:76-8.

6. Coan EB1, Doan A, Allen C. Mucinous eccrine carcinoma: a rare case of recurrence with lacrimal gland extension. Ophthal Plast Reconstr Surg 2012;28:e10910.

7. Breiting L, Christensen L, Dahlstrøm K, et al. Primary mucinous carcinoma of the skin: a population-based study. Int $\mathrm{J}$ Dermatol 2008;47:242-5.

8. Wright J, Font RL. Mucinous sweat gland adenocarcinoma of the eyelid: a clinicopathologic study of 21 cases with histochemical and electron microscopic observations. Cancer 1979;44:1757 